\title{
Deuterium charged grain boundaries in iron investigated at room and cryo temperatures with APT
}

\author{
Martina Heller, Chandra Macauley, Benedict Ott and Peter Felfer \\ FAU Erlangen, Erlangen, United States
}

In the presence of hydrogen $(\mathrm{H})$ donating environments, the strength of high-strength metallic materials is limited by hydrogen-induced embrittlement. Since $\mathrm{H}$ embrittlement is caused by the interaction of $\mathrm{H}$ with crystal defects such as grain boundaries, dislocations and precipitated second phases, a thorough understanding of these interactions is needed. Atom probe tomography (APT) is a suitable method for the quantitative, near-atomic scale investigation of $\mathrm{H}$ at crystal defects, if the $\mathrm{H}$ can be preserved at the crystal defects. For the experiments, we used deuterium-(D) as a tracer for $\mathrm{H}$ to be able to distinguish it from spurious $H$ present in the APT analysis chamber. The bulk sample is charged with D and a site specific lift out in a scanning electron microscope (SEM) in combination with a focused ion beam (FIB) is performed at a grain boundary. The time of measurement in the atom probe a CAMECA LEAP 4000X HR is varied after charging to detect any time difference of the behaviour of $\mathrm{D}$ at the grain boundary, e.g. cluster formation. To investigate the initial state of D at the grain boundary directly after charging, a cryo lift out process is developed. After charging, the sample is transferred into the FIB/SEM that is equipped with a cooling stage that can keep the temperature at $-180^{\circ} \mathrm{C}$. Inside the SEM, the sample preparation continues with a site specific FIB lift-out process of a sample at a grain boundary at cryogenic temperatures to prevent loss of the D. For the lift out process the sample is heated up to $0^{\circ} \mathrm{C}$ to enable Platinum deposition with a gas injection system inside the SEM. For the transfer from the SEM into the atom probe we used a custom designed transfer system that enables cryogenic- or room-temperaturetransfer of atom probe specimens. This system can be used to move specimens between cryogenic electro polishing, coating deposition, FIB milling, a modified commercial CAMECA LEAP 4000X HR, and a newly built titanium APT instrument for the direct analysis of ${ }^{1} \mathrm{H}$. During the transfer, the specimen is kept in an actively cooled transfer arm at a temperature of $-160^{\circ} \mathrm{C}$ in a vacuum. The transfer time between the FIB/SEM and the LEAP are about $30 \mathrm{~min}$. Inside the LEAP in the buffer chamber is a custom made

heating and cooling stage that enables a constant cooling chain during transfer and a precooling of the LEAP sample puck. [1]

In depth analysis of the APT data was conducted with a custom MATLAB@ Toolbox. This Toolbox, which is freely available on GitHub, enables Grain boundary and Cluster analysis as well as a variety of other tools. The results will show that the combination of APT with FIB/SEM at room and cryogenic temperature of deuterium charged iron samples gives a deeper insight on how hydrogen effects metallic materials and will greatly aide in the rational design of hydrogen resistant materials concepts for the transport and storage of $\mathrm{H}$.

\section{References}

[1] Macauley et al., A versatile cryo-transfer system, connecting cryogenic focused ion beam sample preparation to atom probe microscopy, PLOS ONE, 2021 